\title{
Germanica
}

\section{„Laßt uns doch mal wieder einen ,Nazi’ verspeisen": Unverdaute deutsch-jüdische Geschichte bei Barbara Honigmann}

"Let's devour a 'Nazi' again!": Indigested German-Jewish History in Barbara Honigmann's Bilder von A

"Mangeons à nouveau un "nazi" ": une histoire judéo-allemande mal digérée dans l'œuvre de Barbara Honigmann

\section{Lilla Balint}

\section{OpenEdition}

Journals

Édition électronique

URL : http://journals.openedition.org/germanica/3036

DOI : 10.4000/germanica.3036

ISSN : 2107-0784

Éditeur

Université de Lille

Édition imprimée

Date de publication : 30 décembre 2015

Pagination : 83-97

ISBN : 9782913857360

ISSN : 0984-2632

\section{Référence électronique}

Lilla Balint, « „Laßt uns doch mal wieder einen „Nazi” verspeisen“: Unverdaute deutsch-jüdische Geschichte bei Barbara Honigmann », Germanica [Online], 57 | 2015, Online erschienen am: 30 Dezember 2017, abgerufen am 06 Oktober 2020. URL : http://journals.openedition.org/germanica/ 3036 ; DOI : https://doi.org/10.4000/germanica.3036 


\title{
„Laßt uns doch mal wieder einen ,Nazi' verspeisen“: Unverdaute deutsch-jüdische Geschichte bei Barbara Honigmann
}

\author{
Lilla BALINT \\ Vanderbilt University, Nashville
}

In ihrem Essay anlässlich des Todes von Thomas Brasch, dem enfant terrible des ostdeutschen Literaturbetriebs, schreibt Barbara Honigmann, dass dieser ,ziemlich wichtigtuerisch benjaminbrechtmüllermäßig“ herumschwadroniere, und zwar mit Begriffen wie „Produktivität Gesellschaft - Widersprüche - Bedürfnisse"1. Es dürfte keineswegs Honigmanns Absicht gewesen sein, an dieser Stelle ihre eigene ars poetica darzulegen, und doch können ihre Worte über Brasch so ziemlich genau als das Gegenstück zu ihrem eigenen ästhetischen Programm gedeutet werden. In ihrem Anliegen, Geschichte fassen zu wollen, ist sie den Autoren, die sie hier ironisch zu einem Adjektiv zusammenwürfelt, durchaus ähnlich, gleichzeitig bewegt sich Honigmann fernab von theoretischen Gebilden, die von abstrakten Konzepten untermauert sind und getragen werden. Im Vergleich zu Walter Benjamin, Bertolt Brecht und Heiner Müller muten ihre Werke auf den ersten Blick gera-

1. - Barbara Honigmann, „Wie viele sind wir eigentlich noch. Zum Tod von Thomas Brasch", in: Das Gesicht wiederfinden. Über Schreiben, Schriftsteller und Judentum, München, Carl Hanser Verlag, 2006, S. 141-150, hier 145. 
dezu einfach, gar simpel an. Honigmanns Prosa kommt nicht nur ohne große Worte aus, es sind auch immer wieder kleine Geschichten, denen sie sich zuwendet. Mit klein ist hier gemeint, dass ihre Erzählungen tief im Alltag wurzeln, und auch dann diese Haftung an der alltagsweltlichen Realität nicht verlieren, wenn es ihr gerade darum geht ,große“ geschichtliche Zusammenhänge hervorzukehren. Die Kunst von Honigmann besteht nicht zuletzt darin, dass sie zeigt, wie Geschichte als ein Geflecht aus eben jenen „kleinen“ Geschichten entsteht - somit gerade die Relativität von „klein“ und „groß“ unterstreicht - und uns zwingt, diese Wörter in Anführungszeichen zu denken.

Anregung für diese Art des Erzählens dürfte sie sich bei Glückel von Hameln, Rahel Varnhagen und Anne Frank geholt haben, denn wie Honigmann schreibt, ,erzählten [diese] Bagatellen und Geschichten des Tages, von denen sich später herausstellen wird, daß sie die große Geschichte ausmachten“ 2 . Im Gegensatz zu historischen Untersuchungen siehe uns in ihren Erzählungen, so Honigmann, „Geschichte direkt an“. Und um diesen unmittelbareren Zugang zu Geschichte verstärkt hervorzuheben, wiederholt sie noch einmal: „Direkt ins Gesicht“3. Anhand dieser drei Autorinnen entwickelt Honigmann in ihrem Essay Eine ,ganz kleine Literatur" des Anvertrauens was man als ihre Ästhetik des Kleinen bezeichnen könnte. Einerseits ist ihr Anliegen historisch, da es ihr primär darum zu tun ist, das Eigentümliche an der Erzählkunst dieser drei deutsch-jüdischen Frauen zu eruieren. Was sie über ihre jüdische Herkunft hinaus verbindet, sei eine gewisse Strategie des Schreibens, stellt Honigmann fest. Mit Memoire, Brief und Tagebuch wählten sie Gattungen, die fernab von den etablierten Formen literarischen Schreibens liegen und optierten damit auch für eine Art des Schreibens, die nichts Diskursives kennt, kaum abstrakte Begriffe verwendet und sich selten im Allgemeinen verliert. ,[Ihr Schreiben] kommt aus der Anschauung und bleibt bei der Beschreibung" "4. In ihren Ausführungen geht Honigmann eine performative Verbindung mit dieser Art des Erzählens ein: sie bleibt dem Kleinen auch dahingehend verpflichtet, dass sie sich ganz nah an den Texten dieser Autorinnen selbst bewegt. Gleichzeitig überschreitet sie diesen historischen Horizont und gleitet ins Programmatische hinüber. Aufgrund der herauskristallisierten ästhetischen Besonderheiten erschreibt sich Honigmann nämlich eine Genealogie, um damit ihr eigenes schriftstellerisches Selbstverständnis darzulegen.

2. - Barbara Honigmann, „Eine ,ganz kleine Literatur“ des Anvertrauens“, in Das Gesicht wiederfinden. Über Schreiben, Schriftsteller und Judentum, München, Carl Hanser Verlag, 2006, S. 7-30, hier 12.

3. - Ebd., S. 12.

4. - Ebd., S. 11. 
Wenn Honigmann fragt, ,ob ich vielleicht auch zu dieser, ganz kleinen Literatur' des Randes, der unkomfortablen Randposition gehöre"5, identifiziert sie sich mit der Erzählweise, die sie Glückel van Hameln, Rahel Varnhagen und Anne Frank zuschreibt. Gleichzeitig lässt sie eine weitere Facette ihrer Ästhetik des Kleinen aufscheinen. Klein beschreibt nicht mehr nur eine Strategie des Schreibens, sondern zeigt auch den Ort in der Gesellschaft an, von dem aus das Erzählen geschieht: die der Minderheit nämlich. Der Blick, der sich stringent dem Individuum und seiner Alltagswelt zuwendet, ist also einer Schreibposition geschuldet, die sich selbst als minoritär erfährt und definiert. Gerade diese aufgezwungene - und auch internalisierte - Randposition erzeugt einen Blick, der sich einer gewissen Verantwortung nicht entsagen kann. Die Verantwortung, bzw. die ethische Haltung, die diesem Blick innewohnt entsteht gerade aus dem Wissen heraus, dass solche Randpositionen meist als Produkt von großen Ideologien verstanden werden können.

Honigmanns Ästhetik des Kleinen setzt also einen Blick voraus, der nicht von den Höhen solcher Abstrakta wie „Produktivität“, „Gesellschaft“, „Widerspruch“ und „Bedürfnisse“ herabblickt, sondern sich dicht am Individuum bewegt, der Person selbst sozusagen nicht von den Fersen weicht. In den eingangs zitierten Worten Honigmanns über Brasch schwingt gerade der Vorwurf mit, dass bei ihm, laut Honigmann, der Fokus auf die Einzelperson oftmals den größeren gesellschaftlichen Zusammenhängen zum Opfer fällt. Folglich verbirgt sich hinter dem unscheinbaren Wort ,klein“ ein auf den ersten Blick kaum wahrnehmbarer politischer Impetus. Klein widersetzt sich dem Konzeptuellen - will gar nicht mehr als ein Wort sein - denn bereits die Bezeichnung „Konzept“ und die daran gekoppelte Idee von einer Art theoretischen Verdichtung würde das Individuum aus den Augen verlieren. Folgen wir der Logik Honigmanns, dann verleiht gerade diese radikale Konzentration auf das Individuum ihrer Ästhetik des Kleinen eine politische Dimension: denn mit diesem Wort widersetzt sie sich jenen großen Ideologien, die die Geschichte des zwanzigsten Jahrhunderts bestimmten und von deren Nachwirken die Welt von Honigmanns Geschichten maßgeblich geprägt ist ${ }^{6}$.

Geschichte, in ihrer ganz speziellen dialektischen Komplexität wie sie durch den Alltag entsteht und sich zugleich im Alltag nieder-

5. - Ebd., S. 28.

6. - Zum Zusammenhang von Alltag und Politik in Honigmanns Schreiben siehe Karin Neuburgers Aufsatz „Banalität des Alltags - Schreibkunst und politischer Anspruch in Barbara Honigmanns Am Sonntag spielt der Rabbi Fußball", in: Amir Eshel und Yfaat Weiss (Hrsg.), Kurz hinter der Wahrheit und dicht neben der Lüge. Zum Werk Barbara Honigmanns, München, Fink, 2013. Siehe weiterhin das Kapitel „Ästhetik des Alltags“ in Petra Fieros Zwischen Enthüllen und Verstecken: Eine Analyse von Barbara Honigmanns Prosawerk, Tübingen, Niemeyer, 2008. 
schlägt - durchzieht das gesamte Oeuvre Honigmanns. Jedoch verlangt ihre Ästhetik des Kleinen nach einer weiteren Qualifizierung. Nur allzu leicht könnte der Begriff des Kleinen zu der Idee verleiten, es ginge Honigmann um einfache Geschichten, deren Simplizität daher rührt, dass sie abgerundet sind, sozusagen aufgehen, weil sie uns einen auflösenden Schluss bescheren. Die Einfachheit ihrer Sprache, und eine auf Anhieb schlicht erscheinende narrative Struktur ihrer Werke stehen jedoch im Spannungsverhältnis dazu, auf das sich Honigmann immer wieder kapriziert: auf das „Zerbrochene nämlich und Inkohärente“7. In Anlehnung an Heinrich von Kleist, geht es ihr immer wieder um Brüche - kleine und große, alltägliche und entscheidende zugleich - die oftmals nicht aufgelöst werden können, denn wie die Idee des Bruchs nahelegt, kann bereits Entzweites nicht einfach wieder gekittet werden. In ihren Werken treffen häufig Figuren in den verschiedensten Konstellationen aufeinander, deren Konflikte, gegenseitiges Unverständnis, und gelegentlich auch das Scheitern ihres Miteinanders unter anderem auf die grundsätzliche Bedingtheit durch Versenkung in Geschichte zurückzuführen ist.

Vielleicht tritt dieses Zerbrochene in keinem ihrer Werke so deutlich in den Vordergrund wie in Bilder von A. In diesem Roman, der bei dieser Analyse im Mittelpunkt stehen wird, erzählt Honigmann die Geschichte einer kurzlebigen Liebesbeziehung und ihr langes Nachleben. Aus einer zeitlichen Distanz von etwa zwei Jahrzehnten rekapituliert die Ich-Erzählerin, damals eine angehende Dramaturgin, ihre Affäre mit dem fünfzehn Jahre älteren Theaterregisseur A. in der Ostberliner Theaterszene der späten siebziger Jahre. Vor dem Hintergrund der Intimität einer solchen Beziehung tritt nicht nur ihr Scheitern besonders eklatant zutage, gleichzeitig wird der Blick auf das „Unaufgelöste und Unauflösliche" "8, also die möglichen Gründe für das Misslingen gelenkt. In Bilder von A. ist es die stille Wirkungsmacht von deutscher Geschichte über die alltagsweltliche Realität dieser Beziehung, der das Scheitern zuzuschreiben ist - darum soll es hier in erster Linie gehen. Die Relevanz dessen für das übergreifende Thema Essen und Ernährung in der Literatur ergibt sich daraus, dass für die Repräsentation „,om Schiefen“ wie Honigmann formuliert - also den Unstimmigkeiten in dieser Beziehung - Essen und die damit verbundenen Praktiken eine zentrale Rolle spielen. Im ersten Teil dieses Essays wird es ausgehend vom Nichtvorhandensein einer Alltäglichkeit zwischen A. und der Ich-Erzählerin um die genaue Spezifik dieser

7. - Barbara Honigmann, „Das Schiefe, das Ungraziöse, das Unmögliche, das Unstimmige", in: Das Gesicht wiederfinden. Über Schreiben, Schriftsteller und Judentum, München, Carl Hanser Verlag, 2006, S. 151-166, hier 151.

8. - Ebd., S. 151. 
Beziehung gehen, einer Liebe, für die Kunst und Poesie als ausschließliche Identifikationsmomente gelten. Im zweiten Teil wird dieses romantische Liebesideal, das von A. und der Ich-Erzählerin gelebt wird, auf Geschichte hin befragt, um zu ermitteln, warum diese Liebe zu keiner alltäglichen Normalität finden kann.

\section{„Möchtest Du Käse oder Marmelade?“"}

Von Anfang an ist die Beziehung von A. und der Ich-Erzählerin von einer gemeinsamen Verehrung künstlerischer Vorbilder durchdrungen. Zueinander findet das Paar über die Liebe zu Kleist, in dessen Preußenkritik sie ihr eigenes Unbehagen gegenüber dem real existierenden Sozialismus der DDR gespiegelt sehen. Spielerisch nennen sie sich gegenseitig „Prinz Jussuf“" und „Mönch am Meer“ - Kosenamen, die sie den Werken von Else Lasker-Schüler und Caspar David Friedrich entlehnen und in denen sich ihre Bewunderung für eben jene Künstler niederschlägt. Ihre Liebe bewegt sich gänzlich in der ,Sfäre der Poesie, in der alles entschiedener, jede Function höher und lebendiger ist und farbiger in die Augen springt" ${ }^{\text {"9 }}$. Die Tatsache, dass Honigmann dieses Zitat von Novalis kursiv gesetzt ihrem eigenen Text einverleibt, anstatt es durch Anführungsstriche von der Geschichte der Ich-Erzählerin abzugrenzen - es also als ein Zitat zu markieren - unterstreicht die Bedeutung eben jener im weiten Sinne des Wortes verstandenen Poesie für das Selbstverständnis dieser Beziehung.

Zwar bewegt sich die Liebe zwischen A. und der Ich-Erzählerin vom ersten Moment an gewandt und gekonnt in der unendlichen Welt des Geistes, die praktischen Grenzen ihrer Beziehung sind jedoch ganz klar abgesteckt. A. hat eine Frau und zwei Kinder, was die Ich-Erzählerin nicht weiter zu stören scheint, denn sie verkündet mit einer Mischung von Naivität und Stolz: „mein Beruf wird ,Liebhaberin” sein“10. Mit der gemeinsam auserkorenen Losung ,stärker, größer, schöner, leidenschaftlicher, dunkler"11 distanziert sich das Paar von bürgerlich geprägten Liebesvorstellungen und bringt damit auch ihren Widerstand gegen die sinnentleerte Realität der DDR zum Ausdruck. „Dicker“ fügt A. noch zu dieser Reihe von Adjektiven hinzu, da er findet, dass seine Liebhaberin „gut ein Paar Kilo zunehmen könnte“12. Doch A.s Ergänzung ist regelrecht paradox. Nicht nur weil dicker ganz und gar nicht zu den von romantischer Intensität nur so strotzenden Adjektiven passt, sondern auch, weil er mit der Heraufbeschwörung von Essen

9. - Barbara Honigmann, Bilder von A., München, Carl Hanser Verlag, 2011, S. 18-19.

10. - Ebd., S. 19.

11. - Ebd., S. 19.

12. - Ebd., S. 18. 
genau jene Dimension anschneidet, die dieser Beziehung fremd bleiben sollte. Was in dieser Liebe voller Kunst und Poesie nämlich keinen Platz findet ist gerade der Alltag, dessen Nichtvorhandensein Honigmann ganz prägnant in Form von einer Frage auf den Punkt bringt: „Ich glaube, wir wollten beide vermeiden, uns beim Frühstück gegenüberzusitzen und zu fragen, möchtest du Käse oder Marmelade?"13. Statt sich mit profanen Detailfragen wie der allmorgendlichen Ernährung abzugeben, lautet ihre Devise „Nur kein Alltag, sondern nur Poesie! Nur Kleist!“"14.

Honigmann bedient sich bei der Darstellung dieser Liebe einer bewussten Zuspitzung. Indem sie die Beziehung von A. und der Ich-Erzählerin des Alltags, und der damit vermeintlich einhergehenden Banalitäten entlastet, unterstreicht sie die romantisch überhöhte Liebesvorstellung, von der diese Künstlerliebe getragen wird. Der Alltag, der mit diesem Bild des ausbleibenden Frühstücks herbeizitiert wird, ist in Bilder von A. schlichtweg nicht vorhanden. Welche Rolle aber spielt diese Abwesenheit? Diese Frage stell uns vor ein analytisches Problem, denn darüber, was in einem Text nicht vorhanden ist, lässt sich bis auf die Tatsache, dass es nun mal fehlt, nicht besonders viel sagen. Somit muss man sich dem, was abwesend ist, auf Umwegen nähern. Der Rückgriff auf Honigmanns Roman Soharas Reise soll dazu dienen, die Bedeutung von Essen und der damit zusammenhängenden Praktiken herauszuarbeiten, um dann vor diesem Hintergrund zu beleuchten, wie es bei ihr um eine Liebe bestellt ist, in der gerade diese Alltagsdimension fehlt.

Zwei Beispiele aus Soharas Reise beleuchten, wie Honigmann Geschichte nicht nur in Zusammenhang mit Essen bringt, sondern anhand dessen gleichzeitig greifbar macht. Im Mittelpunkt des Romans steht Sohara, eine sephardische Jüdin, die als Jugendliche gezwungen ist, mit ihrer Familie von Algerien nach Frankreich zu flüchten. Zwar ist Frankreich, das ein ,zivilisiertes Land!“ und das „Land der Freiheit!“15 genannt wird, durchaus positiv besetzt, doch die darüber hinausgehende persönliche Erfahrung von Vertreibung - wenn sich Identität sich nicht nur langsam, graduell, und kaum wahrnehmbar wandelt, sondern gezwungen ist, sich schlagartig der verändernden örtlichen Realität anzupassen - ist hier im Koffer versteckt: „Die beiden Koffer waren vor allem mit Tüten und Dosen angefüllt, in denen meine Mutter allerlei Gewürze mit herüberbrachte, von denen sie mit Recht annahm, daß wir sie in Europa nicht so leicht wiederfänden, und die doch ganz unerläßlich für unsere Speisen waren"16. Die Gewürze versinnbildlichen

13. - Ebd., S. 17.

14. - Ebd., S. 17.

15. - Barbara Honigmann, Soharas Reise, München, dtv, 2010, S. 44.

16. - Ebd., S. 40. 
die alte Identität, die nicht abgelegt, sondern in die neue Welt tatsächlich mit hineingetragen wird. Jedoch geht mit der Migration auch eine Verflüchtigung dieser algerischen Identität einher, da sie sich von Oran als konkretem Ort lösen muss, was symbolisch durch die Gewürze zum Ausdruck gebracht wird: Diese können ihre Funktion schließlich erst in der Praxis des Kochens erfüllen. Der Akt der Zubereitung bedeutet allerdings eine im buchstäblichen Sinne verstandene substantielle Transformation - Gewürze büßen ihre Materialität ein, um ihre Wirkung fortan in der ätherischen Sphäre von Gerüchen und Geschmäckern zu entfalten.

Die enge Verknüpfung von Geschichte mit der Nahrungsaufnahme bringt auch ein weiteres Beispiel aus dem Roman zum Ausdruck. Frau Kahn, Soharas aschkenasische Nachbarin in Straßburg, drückt ihre gänzlich säkulare jüdische Identität ebenfalls über das Essen aus. Bei ihr steht weniger die Zubereitung von Speisen im Vordergrund als die Praktiken des Einkaufens. Frau Kahn, die eine „tätowierte Nummer auf ihrem Unterarm“ trägt, hat seit fünfzig Jahren „,kein Deutsch und mit keinem Deutschen mehr gesprochen"17. Zu ihrer ehemaligen Heimat hat die Holocaust-Überlebende ,alle Brücken abgebrochen“"18. Honigmann kehrt die Redewendung vom Symbolischen ins Buchstäbliche, da Frau Kahn selbst den kurzen Weg über den Rhein nach Kehl scheut. Dem Spartourismus trotzend, ,fährt [sie] auch nicht zu Aldi, was sonst alle tun" 19 und weigert sich, ihre leibliche Existenz mit Lebensmitteln aus Deutschland zu sichern, dem Land, das ihr Leben beinahe ausgelöscht hätte. „Ich brauche nichts mehr von denen“, verkündet sie „,auch nicht billiger bei Aldi“ 20 .

In jeweils unterschiedlichen Ausprägungen gehören Speisen und die damit verbundenen Praktiken sowohl für Sohara als auch für Frau Kahn zu identitätsstiftenden Momenten. Die Signifikanz von Essen wird jedoch noch durch einen weiteren, und zwar rituellen Aspekt unterstrichen, der diese Frauen zusammenführt. Das Eis zwischen ihnen bricht nämlich an Sabbat, als Frau Kahn, die als nichtreligiöse Jüdin eigentlich „,keine Notiz davon [nimmt], ob es nun gerade Dienstag, Mittwoch oder Sabbat ist“, Sohara vorschlägt, sich am Freitag „zusammenzutun“21. Frau Kahn bereitet „eine Art gefillte Fisch“ zu, worüber sich Sohara in einem beinahe abfälligen Ton als „,das zweite Heiligtum der Aschkenasim nach den KZs“22 lustig macht und selber

17. - Ebd., S. 22.

18. - Ebd., S. 21.

19. - Ebd., S. 21.

20. - Ebd., S. 22-23.

21. - Ebd., S. 71.

22. - Ebd., S. 72. 
eine Dafina auftischt. Entscheidend ist hierbei, dass diese zwei unterschiedlichen jüdischen Lebenswelten schlussendlich am Tische zusammengeführt werden. An dem gemeinsam begangenen Sabbat steht nicht nur der gefillte Fisch neben der Dafina, sondern im Nebeneinander dieser Speisen manifestiert sich gleichzeitig auch ein Miteinander der Dinierenden. Der gedeckte Tisch symbolisiert einen zustande gekommenen Austausch zwischen der gottesfürchtigen Sohara, die an Sabbat den Telefonstecker zieht, und Frau Kahn, die solche Vorschriften lediglich für „Formalitäten“ hält und Sohara nahe legt - ganz im Sinne von Kants aufklärerischem Diskurs - sich ihrer Vernunft zu bedienen und zu tun, was sie für richtig hältt23.

Jedoch heißt das nicht, dass die beiden Frauen tatsächlich zu einer gemeinsamen Sprache finden würden. „Dass die Seßhaften die Flüchtigen nie verstehen" 24 , wie es Frau Kahn formuliert, verbindet sie zwar, aber ihre Erzählungen von Flucht und Verfolgung sind zu unterschiedlich, als dass sie sich nahtlos zu einer großen Geschichte zusammenfügen ließen. Wie Sohara fremdeln anmerkt „sagt [Frau Kahn] immer ,diese Lager' und ,die Kannibalen', sie hat eine eigene Sprache für ,das' gefunden, weil man ,es' wie sie sagt, sowieso nicht beschreiben kann“25. Für sie bleibt die Geschichte des Holocaust dennoch „eine einzig große Erzählung", oder ,vielmehr eine unheimliche Landschaft" 26. Sowohl der Begriff „Erzählung“ als auch „unheimlich“ heben durch ihren impliziten Verweis auf das Märchenhafte gerade das Fiktionale hervor. Dabei geht es nicht um eine Anzweiflung der Shoah, sondern darum, dass sie für Sohara den Status des Überlieferten nicht überschreiten kann und damit nicht über die Durchschlagskraft der eigens durchlebten Geschichte verfügt.

Mit der Tatsache, dass an dem Sabbat nicht viel gegessen wird, bringt Honigmann genau diese Distanz auf den Punkt. Auch bei diesem Aufeinandertreffen bei Tische bleibt sie also dem Kleinen verschrieben. Da ihre narrative Welt fast nie das verlässt, was man als das wahrscheinlich Mögliche bezeichnen könnte, sind der gefillte Fisch und die Dafina kleine Gesten der Annäherung. In dieser Kapazität sind sie allerdings dem Käse und der Marmelade aus Bilder von A. diametral entgegengesetzt, deren Abwesenheit ja gerade bezeugt, dass A. und die Ich-Erzählerin sich eben nicht an denselben Frühstückstisch setzen, und der Alltag ihrer Beziehung fremd bleibt. An kleinen Gesten fehlt es auch zwischen ihnen nicht, allerdings sind diese gänzlich anderen Charakters. Ihre Beziehung wird fast ausschließlich von extensiven Praktiken der

23. - Ebd., S. 75.

24. - Ebd., S. 72.

25. - Ebd., S. 73.

26. - Ebd., S. 24. 
Kommunikation getragen, die ihre hochkulturelle Anbindung nur selten verlieren:

Manchmal war es auch nur ein Zettel, manchmal ein Liebeswort, manchmal ein böses Wort, manchmal eine Kunstpostkarte, an der wir noch weitergezeichnet hatten und die dadurch alle möglichen Anspielungen und Botschaften erhielt, manchmal ein paar Zeilen aus einem Gedicht, ein aufgeschnapptes Wort oder eine ganze Seite aus einem Buch, der Text eines Dichters, den man besser in sich eindringen lassen konnte, wenn man ihn mit der Hand abschrieb, weil er Worte enthielt, in denen wir wiederfanden, was wir dachten und fühlten ${ }^{27}$.

A. und die Ich-Erzählerin verharren also in der Sphäre der Poesie. „Personen die keinerlei spezielles Interesse teilen,“ schreibt Georg Simmel, „können sich bei dem gemeinsamen Mahle finden“ 28 . Geben wir Simmel recht, dass sich sogar gänzlich wesensunähnliche Menschen beim Speisen näher kommen können, ist es umso auffälliger, wenn sich Personen nicht an einen Tisch setzen, die sonst durch poetische Kommunikationspraktiken verbunden sind. Der Zusammenhang stellt sich wie folgt dar: Ohne sich des Zynismus verdächtig $\mathrm{zu}$ machen, kann man davon ausgehen, dass diese Art von intensivem, geistigem Austausch Beziehungen längerfristig nur dann inne wohnt, wenn es ihnen verwehrt bleibt, in eine Art von Normalität überzugehen - genau jenen Alltag zu etablieren, für den hier Käse und Marmelade stehen. In seiner soziologischen Untersuchung zur Liebe formuliert es Niklas Luhmann ähnlich. Ein „System von Intimbeziehungen“, schreibt Luhmann, baue sich ,eine eigene, konkrete Welt“, um dann von dieser Welt getragen $\mathrm{zu}$ werden, aber gerade dadurch der Passion entraten könne ${ }^{29}$. Der Zustand von emphatischer Kommunikation kann also nur erhalten bleiben - Passion erlischt nicht, um es mit Luhmann auszudrücken - weil es dieser Beziehung gerade nicht gelingt, eine gemeinsame Welt zu etablieren, die von Alltagspraktiken zusammen gehalten wird.

In Bilder von $A$. findet eine Umcodierung von der romantischen Liebesvorstellung statt. Diese Idee, die im Literarischen überwiegend positiv besetzt ist, gewinnt angesichts der bereits etablierten Bedeutung des Alltäglichen bei Honigmann einen melancholischen Unterton. Denn Alltäglichkeit wird bei ihr nicht als defizitär gedacht, als ein Zustand also, in dem es an etwas - so etwa an Passion - mangelt. Vielmehr stellt in ihrer narrativen Welt Alltag etwas dar, dessen Vorhandensein nicht

27. - Barbara Honigmann, Bilder von A., a.a.O., S. 9-10.

28. - Georg Simmel, "Die Soziologie der Mahlzeit", in: Soziologische Ästhetik (herausgegeben von Klaus Lichtblau), Wiesbaden, Verlag für Sozialwissenschaften, S. 155-161, hier 155 .

29. - Niklas Luhmann, Liebe. Eine Übung, Frankfurt a.M., Suhrkamp, 2008, S. 58. 
vorausgesetzt werden kann und dementsprechend eine Aufwertung erfährt. Alltäglichkeit muss erschaffen werden, was erstens heißt, dass dieses Unterfangen sehr wohl Scheitern kann, wie es in Bilder von A. auch demonstriert wird. Zweitens kann diese alltägliche Normalität nur unter bestimmten Umständen entstehen. Vor diesem Hintergrund erscheint gerade die romantisch geprägte Vorstellung von Liebe als unzulänglich, denn die Beständigkeit ihrer Intensität erlangt sie dadurch, dass sie Käse und Marmelade als Gegenstand gemeinsamen Erlebens nicht gelten lässt. Daraus ergibt sich die entscheidende Frage, warum das gemeinsame Frühstücken, eine Alltäglichkeit in Bilder von $A$. nicht etabliert werden kann.

\section{Poesie vs. Geschichte}

Das Nichtvorhandensein eines Alltags und das Scheitern dieser Beziehung erlangt vor dem Hintergrund der Familiengeschichten von A. und der Ich-Erzählerin eine ganz andere Bedeutung. Wer sich hier nicht an einen Frühstückstisch setzt, also die Sphäre der Häuslichkeit nicht miteinander teilt, ist der Sohn eines bei Stalingrad gefallenen Wehrmachtsoldaten und die Tochter jüdischer Eltern, die dem Holocaust durch ihr Londoner Exil entkamen. Das ausbleibende Frühstücken ist also symbolisch aufgeladen: Die Abwesenheit eines gemeinsamen Alltags wird implizit auf geschichtliche Gründe zurückgeführt. Honigmann zeichnet hierbei die Wirkungsmacht von Geschichte nach, ihr Vermögen bis in die kleinsten, noch so unwesentlich anmutenden Details der Gegenwart vorzudringen, dabei den Status von etwas Gewesenem zu verlieren und sich in etwas Vorhandenes zu verwandeln.

Bereits die erste Begegnung von A. und der Ich-Erzählerin ist mit Herkunft und Geschichte durchwoben:

A. war gerade von seiner ersten West-Inszenierung zurückgekehrt [...]. In München, vielleicht war es auch eine andere Stadt, hatte er Die Wupper von Else-Lasker Schüler inszeniert [...]. Er stand noch ganz im Banne dieser Entdeckung und sei, wie er mir später sagte, nicht nur über mein Grinsen schockiert gewesen, sondern darüber, wie ähnlich ich der Lasker-Schüler sähe, so schwarz und dunkeläugig. Offensichtlich wußte er nicht, oder wollte es auch nicht wissen, daß deutsche Juden oft so aussehen [...]. ${ }^{30}$

Wie so oft bei Honigmann, steckt der Teufel auch hier in den Details. Als Theaterregisseur müsste A. eigentlich literaturgeschichtlich bewandert sein und um die jüdische Herkunft Else Lasker-Schülers wissen. Weitaus verblüffender als die Unkenntnis von A. ist jedoch die

30. - Barbara Honigmann, Bilder von A., a.a.O., S. 13. 
Ungewissheit der Ich-Erzählerin, die sich in dem Wort „oder“ manifestiert (,Offensichtlich wußte er nicht, oder wollte es auch nicht wissen“ meine Hervorhebung). Zwischen nicht wissen und nicht wissen wollen gibt es schließlich einen erheblichen Unterschied. Während Ersteres von schierer Ignoranz zeugt, setzt Letzteres voraus, dass man um etwas sehr wohl weiß, aber es ganz bewusst ignoriert, dem Nichtwissen also eine Entscheidung vorausgeht. Die Tatsache, dass die Ich-Erzählerin in dieser, besonders entscheidenden Frage ihren Mutmaßungen überlassen ist, eröffnet einen für unsere Analyse wichtigen Wesenszug dieser Beziehung: Die Verbindung von A. und der Ich-Erzählerin mag sich zwar fortwährend in und durch Poesie ausdrücken, doch über Geschichte, um noch genauer zu sein, über die deutsche Geschichte des zwanzigsten Jahrhunderts wird in dieser Beziehung geschwiegen. Dieses Schweigen klingt bereits in dem soeben zitierten ersten Gespräch zwischen ihnen an. Statt direkt auf A.s genuine oder gewählte Ignoranz zu reagieren, weicht die Ich-Erzählerin aus, und berichtet ihm, dass sie tatsächlich mit der Else Lasker Schüler verwandt ist. Damit nennt sie den Grund für ihre Ähnlichkeit mit der Dichterin, ohne ihn wirklich zu benennen: dass sie beide jüdischer Herkunft sind, bleibt unangesprochen.

Auch A.s Herkunft wird zwischen den beiden nie explizit thematisiert. Dabei steht den zwei Liebenden Geschichte regelrecht ins Gesicht geschrieben. Schließlich ist es kein Zufall, dass diese Szene des ersten Erblickens auch umgekehrt stattfindet - und somit hervorgehoben wird. Der Blick auf das Äußere von A. wird uns durch die Mutter der Ich-Erzählerin gewährt, die sich vor Entrüstung kaum zurückhalten kann: „Was für ein blonder, blauäugiger Gewittergoi, so ein richtiger Germane, Teutone, Ostgote, und auch noch Preuße“, ruft sie entsetzt ${ }^{31}$. Die plakative Hervorhebung des Aussehens vom stereotypisch deutschen A. und der Ich-Erzählerin, die hingegen vom ,dunklen Typ“ ist, bewirkt, dass ihr Schweigen über Geschichte umso schwerer wiegt. Die optische Markierung ihrer unterschiedlichen Herkunft steigert den Effekt der Stille, denn somit wird über etwas nicht gesprochen, was sichtlich im (narrativen) Raum steht.

Das Bedrückende an ihrem Schweigen wird dadurch intensiviert, dass an keiner Stelle erklärt wird, wie dieses diskursive Vakuum eigentlich entsteht. A. und die Ich-Erzählerin handeln Geschichte nicht als Tabu aus, vielmehr scheint es von vornherein ein implizites Abkommen zwischen ihnen zu geben, welche Themen es zu meiden gilt. Mit implizit ist hier gemeint, dass nicht einmal darüber geredet werden muss, worüber nicht geredet werden soll. Die Selbstverständlichkeit die stillschweigende Übereinkunft -, mit der A. und die Ich-Erzählerin

31. - Ebd., S. 36. 
Herkunft und Geschichte von Anfang an ignorieren, wird somit dem breiteren kommunikativen Kontext zugeschrieben, der ihrer Beziehung vorausgeht und in den sie eingebettet sind. Erst dieses gesellschaftlich angelegte Schweigen ermöglicht es ihnen, Geschichte von vornherein aus dem Weg zu gehen, ihr Existieren, das ja sichtlich zwischen ihnen steht, einfach zu ignorieren. Von diesem diskursiven Tabu her kann auch die Unbeholfenheit der Ich-Erzählerin gedeutet werden, die Eigenart ihrer Beziehung mit A. zu fassen:

Von Anfang an waren wir uns immer gleichzeitig zu nah und zu entfernt, wußten nicht, wie wir es sagen, wie wir es ertragen sollten, und fühlten und sowohl verloren in der Zeit und am Ort, jeder aus seinen Gründen, aber festhalten konnten wir uns nirgends und am wenigsten aneinander. Irgend etwas zwischen uns wog zu schwer und hörte nicht auf, an uns zu zerren. ${ }^{32}$

Was in dieser Passage nicht konkret benannt wird, weil die Ich-Erzählerin an dieser Stelle auf ihr einstiges Selbst zurückgreift, das aufgrund seiner Involviertheit in der Beziehung ihre Dynamik nicht ergründen konnte, ist genau die gesellschaftliche Bedingtheit des Privaten. Erst im Laufe des Briefwechsels, das die Beziehung um etliche Jahre überdauern sollte, wird der Ich-Erzählerin allmählich klar, dass dieses gewisse Etwas, das so schwer zwischen ihnen wog, unter anderem mit ihren unterschiedlichen Geschichten zu tun hatte.

Ihre Affäre reißt zwar mit A.s plötzlicher Emigration in den Westen ab, aber beinahe bis zu A.s Tod, etwa zwei Jahrzehnte später, schreiben sie sich Briefe. „Unsere Wege haben sich voneinander entfernt, und wir sind doch verbunden geblieben“, stellt die Ich-Erzählerin fest, „unauflöslich und unerlöst in einem Ich-weiß-nicht-Was"33. Im langen Nachspiel zu ihrer kurzen Beziehung zeichnet sich die Geschichte einer Entfremdung ab, die wohl bereits in ihrer Affäre angelegt war. Was sich in ihrem Schriftverkehr vollzieht ist nicht nur eine Distanzierung, ausgelöst durch die Hinwendung der Ich-Erzählerin zum Judentum, was bei A. nicht nur auf Unverständnis, sondern gar auf direkte Ablehnung stößt und auch offen von ihm missbilligt wird: „Was soll das, warum heiratest du? Und jüdisch. Wozu?"34, fragt er die Ich-Erzählerin vorwurfsvoll. Nach und nach rückt diese sich auftuende und immer breiter werdende ideologische Kluft zwischen ihnen auch die romantische Liebe von einst - samt ihrer tragenden Idee und kommunikativer Praktiken - in ein gänzlich anderes Licht. Der retrospektive Blick der Ich-Erzählerin enthebt diese von romantischer Übersteigerung geprägte Liebe allmäh-

32. - Ebd., S. 11.

33. - Ebd., S. 9.

34. - Ebd., S. 91. 
lich aus ihrer klaustrophobischen Privatheit und befragt sie auf ihre gesellschaftlichen Konstituenten hin. Rückblickend erscheint Poesie nicht mehr als reiner Ausdruck hochkultureller Affinität, sondern als eine wenn auch unbewusste Strategie der Vergangenheitsvermeidung.

Die Geschichte des Nationalsozialismus und des Zweiten Weltkriegs wird zwar wiederholt angerissen, jedoch bezeichnender Weise in Form eines Tabus: „Ab und zu schlug entweder mein Vater oder A. vor, laßt uns doch mal wieder einen "Nazi' verspeisen“35. Der Aufruf forderte dazu auf, dem Restaurant „Ganymed“" wieder einen Besuch abzustatten, ,dem einzigen Restaurant in Ostberlin, wo es ein chinesisches oder sonst irgendwie ostasiatisches Gericht gab, oder das wenigstens so tat und so hergerichtet war, als ob, und unter dem Namen ,Nasi Goreng' serviert wurde“36. Die Aufforderung entwickelt sich also aus einem Wortspiel, weil sich Nazi auf Nasi Goreng37, dem asiatischen Reisgericht, reimt. ${ }^{38}$ Folgt man Sigmund Freuds Ausführungen zum Witz und seine Beziehung zum Unbewussten, so scheint dieser spezifische Fall des Wortspiels seine Grundthese zu bestätigen: dass Witze zwar zum Lachen bringen, deswegen aber keineswegs harmlos sind. 39 Wortspiele erlauben, so Freud, dass wir „unsere psychische Einstellung auf den Wortklang anstatt auf den Sinn des Wortes zu richten“40. Auf die Geschichte des Dritten Reichs und den Nationalsozialismus wird

35. - Ebd., S. 38.

36. - Ebd., S. 38.

37. - Es dürfte wohl dem Zufall zuzuschreiben sein, dass Nasi Goreng ein Gericht ist, dass sowohl gekocht als auch gebraten wird (der Reis muss vor dem Anbraten bereits vorgekocht sein). Als Wortspiel, das auf das Menschenfressen verweist - genauer gesagt auf das Nazifressen - vereint Nasi Goreng damit zwei Arten des Kannibalismus. In seinem Aufsatz „Das kulinarische Dreieck“ unterscheidet Claude Lévi-Strauss zwischen „Endo-Kannibalismus“ und „Exo-Kannibalismus“. Ersteres bezieht sich auf Verwandte, die eher gekocht werden, letzteres auf Fremde bzw. Feinde, die gebraten werden, womit der Kannibalismus - laut Lévi-Strauss - genau der gleichen Zweiteilung unterliegt wie die sonstige Nahrungszubereitung. Dass Nasi Goreng, das dem Wortspiel gemäß für Nazis steht, diese Dichotomie unterläuft deutet gerade darauf hin, dass Nazis weder in die eine, noch in die andere Kategorie fallen, also weder fremd noch verwandt sind. Zum Kannibalismus siehe Claude Lévi-Strauss, „The Culinary Triangle“, in: Food and Culture: A Reader. New York, Routledge, 2013, S. 40-48, hier 42-43.

38. - Das Restaurant „Ganymed“" gab es in Ostberlin tatsächlich. Ein Blick in die Tagespresse der DDR zeigt weiterhin, dass hier auch das Gericht Nasi Goreng serviert wurde. So etwa schreibt die „Die Neue Zeit“ in ihrer Samstagsausgabe vom 27. Mai 1972: „Ob Nassi [sic] Goreng oder Amsterdamer Kirschsteak, Bongé [der Küchenchef] sagt ,bon' und das bedeutet, daß einem ein Genuß sicher ist [...] Im Ganymed, dem Treff der Schauspieler und Ärzte der nahen Charité, ist ein Hauch von Weltniveau auf der Zunge zu spüren.“ Jahrgang 28, Ausgabe124, Seite12.

39. - Siehe Freuds Aufsatz "Der Witz und seine Beziehung zum Unbewussten".

40. - Sigmund Freud, Der Witz und seine Beziehung zum Unbewussten, Leipzig und Wien, Franz Deuticke, 1905, S. 100. 
durch das Tabu des Kannibalismus lediglich angespielt. Pflichtet man jedoch Freud bei, dann wird der Effekt des Nichtaussprechens durch das Wortspiel „Nazi-Nasi“ noch auf eine andere Ebene gehoben, da die akustische Qualität weiter vom eigentlichen Inhalt (Nazi) ablenkt.

Dem Aufruf „Laßt uns doch mal wieder einen Nazi verspeisen!“ wohnt die Geste der Grenzverletzung im mehrfachen Sinne inne. Erstens fordert der Satz ganz unverblümt dazu auf, mit einem der ältesten Tabus zu brechen, dem Menschenfressen nämlich. Zweitens bedienen sich die Figuren bei ihrer Aufforderung zum Kannibalismus eines heiter-ironischen Tons, der nur deswegen als legitim erscheint, weil es sich bei dem Gegenstand des Verspeisens um Nazis handelt. Der humoristische Effekt, den der Satz angelegt ist zu erzeugen, sollte jedoch nicht darüber hinwegtäuschen, dass ihm eine ganz spezifische - und zwar ernste - Logik zugrunde liegt: es ist der Tabubruch, über den hier eine Verbindung zwischen den Naziverbrechen und dem Kannibalismus hergestellt wird. ${ }^{41}$ Indem Honigmann auf die nonverbale Praxis des Essens verweist, genauer gesagt auf die verbotene Praxis des Menschenfressens, erteilt sie eine radikale Absage an verbale Diskurse über die Vergangenheit. Fast ist man gewillt zu sagen, dass damit das Paradigma der Vergangenheitsbewältigung, das ja gar nicht anders kann, als sich einer extensiven Verbalität zu bedienen, entmachtet wird. Der Satz scheint nahe zu legen, dass eine Antwort auf die Naziverbrechen genau im Nazifressen bestehen würde - einem Akt also, der nicht vollzogen werden kann, gerade weil Kannibalismus ein Tabu darstellt.

\section{Schlussbetrachtungen}

Die Dramatik von deutsch-jüdischen Beziehungen nach dem Holocaust spielt sich zwischen der nicht gestellten Frage „Möchtest Du Käse oder Marmelade?“ und dem Aufruf „Laßt uns doch mal wieder einen Nazi verspeisen“ ab. Durch den Verweis auf das Frühstücken in trauter Zweisamkeit legt Ersteres eine Normalität nahe, die in Bilder von A. nicht etabliert wird. Ein Alltag von Käse und Marmelade kann auch nicht einkehren, impliziert der Roman, gerade weil Letzteres, die Aufforderung zum Tabubruch, ebenfalls im kommunikativen Raum existiert. Normalität wird durch ein schleichendes und doch deutlich präsentes Unbehagen verhindert, das sich in Form dieses wiederkehrenden Wortspiels immer von neuem entlädt. A. und die Ich-Erzählerin schaffen es nicht, das Schweigen über ihre jeweilige Familiengeschichte, also über ein Stück deutsch-jüdische Geschichte zu durchbrechen. Auch in ihrem jahrzehntelangen Briefwechsel spre-

41. - Ganz explizit wird der Zusammenhang in Soharas Reise hergestellt, wo Frau Kahn die Deutschen wiederholt als Kannibalen bezeichnet, siehe S.23. 
chen sie ihre Herkunft nicht direkt an. Geschichte macht sich hier im Unausgesprochenen bemerkbar, im expliziten Nichtsprechen darüber und in der allmählichen Entfremdung von A. und der Ich-Erzählerin. Bezeichnenderweise ist es ein fiktives Gespräch über Judentum und jüdisch sein, das in den Text mit einfließt. Die Ich-Erzählerin stellt sich nach A.s Tod die Unterhaltung vor, die sie nie geführt haben und Worte, die sie „nie gesagt haben“42. Am Ende dieser „Disputation“, wie es die Ich-Erzählerin formuliert, legt sie A. folgende Worte in den Mund: „Wie du redest! Ich erkenne dich nicht. Wie peinlich! Ich kann dir gar nicht sagen, wie peinlich ich das alles finde“43.

Mit den zwei auf das Essen bezogenen Sätzen reißt Honigmann einen Diskurs an, der seit dem Ende des Zweiten Weltkriegs immer wieder Thema öffentlicher Diskussion ist: Wie kann man mit der nationalsozialistischen Vergangenheit umgehen? In Bilder von A. skizziert Honigmann ein deutsch-jüdisches Zusammenleben, das nicht zustande kommt, eine Beziehung, in der es keine alltägliche Normalität gibt, sondern die sich fast fluchtartig in die Sphäre der Hochkultur begibt. Poesie wird bei Honigmann zum Verdrängungsmechanismus, die Konzentration auf das Geistige, bzw. ausschließlich darauf, ermöglicht es einer Konfrontation mit Geschichte aus dem Weg zu gehen. An dieser Stelle scheint eine Kritik Narrativen gegenüber durch, die sich alleinig auf die Sphäre der Hochkultur - auf die sogenannten „Kulturleistungen“ - in der deutsch-jüdischen Geschichte kaprizieren, ohne den gesellschaftlich-historischen Hintergrund mit einzubeziehen. In ihrer Kritik geht Honigmann jedoch einen entscheidenden Schritt weiter. Denn der spielerische Aufruf zum Naziverspeisen - einer Aktivität also, die das Diskursive hinter sich lässt - legt eine zweifache Skepsis bezüglich des unendlichen Dialogs über Geschichte nahe: nicht nur die Adäquatheit von Vergangenheitsbewältigung wird hier angezweifelt, sondern auch die Sinnhaftigkeit dieses Paradigmas. Deutsch-jüdische Geschichte bleibt unverdaut, gar unverdaubar. 
